\title{
The benign tumours of skin adnexal diagnosed in ouagadougou: Histopathological and epidemiological profile
}

\section{Aimé Sosthène Ouédraogo ${ }^{1,2}$, Norbert W Ramdé1,2, Muriel Sidnoma Ouédraogo $0^{2,3}$, Lamien-Sanou Assita, ${ }^{1,2}$, Franck A. H. A Ido', Ibrahim Savadogo, Souleymane Ouattara5, Olga Mélanie Lompo ${ }^{1,2}$}

${ }^{1}$ Department of Pathology, Yalgado Ouédraogo University Teaching Hospital of Ouagadougou, Burkina Faso, ${ }^{2}$ University of Ouaga 1 Pr Joseph KI-ZERBO, Burkina Faso, ${ }^{3}$ Division of Dermatology and Venerology, Yalgado Ouédraogo University Teaching Hospital of Ouagadougou, Burkina Faso, ${ }^{4}$ Department of Pathology Regional Hospital of Ouahigouya, Burkina Faso, ${ }^{5}$ Department of Pathology, Blaise Compaoré University Teaching Hospital Burkina Faso

Corresponding author: Dr. Aimé Sosthène Ouédraogo, E-mail: sostheneaime@yahoo.fr

\begin{abstract}
Introduction: The tumours of skin adnexal are rare and very often benign. Because of their large diversity they caused some diagnose and classification problems. They are tumours of ten mixed up with other skin tumours. The objective of this study was to study the histopathological and epidemiological profile of the benign skin adnexal tumours in Ouagadougou and to classify them according to the differenciation type in order to well know them and improve their treatment. Methodology: We conducted a retrospective study on the period of sixteen years going from $1^{\text {st }}$ January 1998 to 31 December 2013. This study interested all cases of benign skin adnexal tumours histologically confirmed (diagnosed) in the three pathology laboratories of the Ouagadougou town. Results: We collected sixty cases of skin adnexal tumours on the total of 763 cases of skin tumours representing $7.8 \%$. The benign tumours represented $86 \%$ of the adnexal tumour cases. These tumours were occurred in the relatively young age and were preferentially located on the head (cephalic) extremity. On the histological plan, the hair follicle tumours were more frequent (42.5\%) followed by sweat gland tumours (30.5\%) and sebaceous gland tumours (27\%). Conclusion: The annex tumours are majoritary benign occurring often on the head extremity and dominated by the hair follicle tumours.
\end{abstract}

Key words: Tumours (tumors); Skin adnexal; Histology; Ouagadougou

\footnotetext{
How to cite this article: Ouédraogo AS, Ramdé NW, Ouédraogo MS, Assita L-S, Ido FAHA, Savadogo I, Ouattara S, Lompo OM. The benign tumours of skin adnexal diagnosed in Ouagadougou : Histopathological and epidemiological profile. Our Dermatol Online. 2017;8(4):393-398

Submission: 01.04.2017; Acceptance: 01.07.2017

DOI: 10.7241 /ourd.20174.112
} 


\title{
Les tumeurs bénignes des annexes cutanées diagnostiquées à ouagadougou: Profil épidémiologique et histopathologique
}

\section{Aimé Sosthène Ouédraogo,,2, Norbert W Ramdé1,2, Muriel Sidnoma Ouédraogo $0^{2,3}$, Lamien-Sanou Assita ${ }^{1,2}$, Franck A H A Ido', Ibrahim Savadogo ${ }^{4}$, Souleymane Ouattara ${ }^{5}$, Olga Mélanie Lompo ${ }^{1,2}$}

\begin{abstract}
${ }^{1}$ Department of Pathology, Yalgado Ouédraogo University Teaching Hospital of Ouagadougou, Burkina Faso, ${ }^{2}$ University of Ouaga 1 Pr Joseph KI-ZERBO, Burkina Faso, ${ }^{3}$ Division of Dermatology and Venerology, Yalgado Ouédraogo University Teaching Hospital of Ouagadougou, Burkina Faso, ${ }^{4}$ Department of Pathology Regional Hospital of Ouahigouya, Burkina Faso, ${ }^{5}$ Department of Pathology, Blaise Compaoré University Teaching Hospital Burkina Faso
\end{abstract}

Corresponding author: Dr. Aimé Sosthène Ouédraogo, E-mail: sostheneaime@yahoo.fr

\begin{abstract}
RESUME
Introduction: Les tumeurs des annexes de la peau sont rares et très souvent bénignes. Elles posent du fait de leur grande diversité des problèmes de diagnostic et de classification. Ce sont des tumeurs souvent confondues avec d'autres tumeurs cutanées. Cette étude avait pour but d'étudier le profil épidémiologique et histopathologique des tumeurs bénignes des annexes cutanées à Ouagadougou et de les classifier en fonction du type de différenciation afin de mieux les connaître et d'améliorer leur prise en charge. Méthodologie: Nous avons mené d'une étude rétrospective sur seize ans, allant du ler Janvier 1998 au 31 Décembre 2013. Cette étude a intéressé tous les cas de tumeurs bénignes des annexes de la peau histologiquement confirmées dans les trois laboratoires d'anatomie pathologique de la ville de Ouagadougou. Résultats: Nous avons colligé soixante cas de tumeurs des annexes cutanées sur 763 cas de tumeurs cutanées soit 7,8\%. Les tumeurs bénignes constituaient 86,7\% des cas de tumeurs annexielles. Ces tumeurs survenaient à un âge relativement jeune et étaient localisées préférentiellement sur l'extrémité céphalique. Sur le plan histologique, les tumeurs pilaires étaient les plus fréquentes (42,5\%), suivies des tumeurs sudorales (30,5\%) et des tumeurs sébacées (27\%). Conclusion: Les tumeurs annexielles sont majoritairement bénignes, survenant souvent sur l'extrémité céphalique et dominées par les tumeurs pilaires.
\end{abstract}

Mots clés: Tumeurs; Annexes cutanées; Histologie; Ouagadougou

\section{INTRODUCTION}

Les tumeurs annexielles cutanées sont des tumeurs développées à partir des annexes de la peau à savoir les follicules pileux, les glandes sébacées et les glandes sudorales. Ce sont des tumeurs le plus souvent bénignes [1]. La grande diversité des types histologiques pose parfois des difficultés diagnostiques et de classification. La littérature sur les aspects histologiques de ces tumeurs au Burkina Faso, est rarissime. Nous avons entrepris cette étude dont le but est d'étudier les particularités histopathologiques de ces tumeurs, de les classifier en fonction du type de différenciation afin de permettre leur meilleur connaissance.

\section{MATÉRIELS ET MÉTHODES}

Nous avons mené une étude rétrospective sur une période de 16 années consécutives, allant du $1^{\text {er }}$ janvier 1998 au 31 décembre 2013. Nous avons analysé tous les diagnostics histologiques de tumeurs annexielles cutanées posés dans les trois laboratoires d'anatomie pathologique de la ville de Ouagadougou. Ces

\footnotetext{
How to cite this article: Ouédraogo AS, Ramdé NW, Ouédraogo MS, Assita L-S, Ido FAHA, Savadogo I, Ouattara S, Lompo OM. Les tumeurs bénignes des annexes cutanées diagnostiquées à Ouagadougou : profil épidémiologique et histopathologique. Our Dermatol Online. 2017;8(4):393-398.

Submission: 01.04.2017; Acceptance: 01.07.2017

DOI: 10.7241 /ourd.20174.112
} 
laboratoires étaient les seuls en activité au Burkina Faso durant la période étudiée. Ils recevaient des prélèvements de patients provenant de toutes les régions du pays. Ont été retenus tous les comptesrendus d'examen anatomo-pathologique concernant les patients de tout sexe et de tout âge.

Tous les prélèvements analysés dans les trois laboratoires ont été fixés par du formol tamponné à $10 \%$. Après inclusion en paraffine, coupes de 2 à 4 microns et étalement sur lames, les pièces ont été colorées à l'hématéine et éosine. Le safran était ajouté en fonction de sa disponibilité dans le laboratoire.

Les tumeurs annexielles ont été réparties en trois grands groupes: un premier groupe constitué des tumeurs à différenciation sudorale; un deuxième groupe rassemblant les tumeurs à différenciation sébacée; le troisième groupe était constitué des tumeurs à différenciation pilaire. Nous avons analysé les informations sociodémographiques des patients concernés, les localisations tumorales, et les données histologiques.

\section{RÉSULTATS}

\section{Fréquence Globale}

Au cours de la période d'étude, nous avons colligé 763 cas de tumeurs cutanées dont 60 cas de tumeurs annexielles représentant $7,8 \%$ de l'ensemble des tumeurs de la peau.

Parmi les 763 cas de tumeurs cutanées, 262 (34,3\%) étaient bénignes et $501(65,7 \%)$ malignes.

Les tumeurs annexielles bénignes avec 52 cas représentaient 19,8\% des tumeurs bénignes cutanées.

Les tumeurs annexielles cutanées étaient constituées de $86,7 \%$ de tumeurs bénignes et $13,3 \%$ de tumeurs malignes.

\section{Les Types Histologiques}

Le tableau suivant montre la répartition des tumeurs bénignes des annexes cutanées selon la différenciation et le type histologique (Tableau 1-7).

Nous avons observé 2 cas d'adénomes tubulo-papillaire chez deux adultes de 20 et 30 ans localisés au membre supérieur et au tronc, 1 cas d'hidradénome tubulopapillifère chez un adulte de 20 ans localisé au membre
Tableau 1 : Répartition des tumeurs bénignes selon le type histologique $(\mathrm{n}=52)$

\begin{tabular}{|c|c|c|c|}
\hline $\begin{array}{l}\text { Type de } \\
\text { differentiation }\end{array}$ & $\begin{array}{l}\text { Type } \\
\text { histologique }\end{array}$ & Effectif & Pourcentage (\%) \\
\hline \multirow[t]{3}{*}{ Pilaire } & Pilomatricome & 09 & 17,3 \\
\hline & $\begin{array}{l}\text { Kyste trichilemmal } \\
\text { proliférant }\end{array}$ & 07 & 13,5 \\
\hline & Trichoépithéliome & 06 & 11,5 \\
\hline \multicolumn{4}{|l|}{$n=22(42,3 \%)$} \\
\hline \multirow[t]{2}{*}{ Sébacée } & $\begin{array}{l}\text { Hamartome } \\
\text { verruco-sébacé }\end{array}$ & 08 & 15,4 \\
\hline & Adénome sébacé & 03 & 5,8 \\
\hline$n=14(27 \%)$ & $\begin{array}{l}\text { Hyperplasie } \\
\text { sébacée }\end{array}$ & 03 & 5,8 \\
\hline \multirow[t]{3}{*}{ Sudorale } & $\begin{array}{l}\text { Porome eccrine } \\
\text { classique }\end{array}$ & 06 & 11,6 \\
\hline & $\begin{array}{l}\text { Hidradénome } \\
\text { nodulaire }\end{array}$ & 03 & 5,8 \\
\hline & Syringome & 02 & 3,8 \\
\hline eccrine & $\begin{array}{l}\text { Cystadénome } \\
\text { eccrine }\end{array}$ & 01 & 1,9 \\
\hline \multicolumn{4}{|l|}{$n=12(23 \%)$} \\
\hline \multirow[t]{2}{*}{ Sudorale } & $\begin{array}{l}\text { Adénome } \\
\text { tubulo-papillaire } \\
\text { apocrine }\end{array}$ & 02 & 3,8 \\
\hline & $\begin{array}{l}\text { Syringocystadénome } \\
\text { papillifère }\end{array}$ & 01 & 1,9 \\
\hline $\begin{array}{l}\text { apocrine } \\
n=04(7,7 \%)\end{array}$ & $\begin{array}{l}\text { Hidradénome } \\
\text { tubulo-papillifère }\end{array}$ & 01 & 1,9 \\
\hline Total & & 52 & 100,0 \\
\hline
\end{tabular}

Tumeurs bénignes pilaires

L'âge

inférieur et 1 cas syringo-cystadénome papillifère chez un adulte de 40 ans localisé au membre supérieur.

\section{DISCUSSION}

Les tumeurs des annexes cutanées sont relativement rares. Cette étude a retrouvé une fréquence de 7,8\% de l'ensemble des tumeurs cutanées. Ce constat est partagé par d'autres auteurs africains et dans le monde [1,2]. En effet Samaïla au Nigéria [1] a retrouvé dans sa série une fréquence de $0,9 \%$. Ce sont des tumeurs à majorité bénignes. Dans notre étude, les tumeurs bénignes ont représenté 86,7\% et les tumeurs malignes $13,3 \%$. Samaila, Radhika et col, Rajalakshm et col ont retrouvé respectivement, $88,5 \%, 77,14 \%$ et $90,48 \%$ de tumeurs bénignes contre $11,5 \%, 22,63 \%$ et 9,52\% de tumeurs malignes dans leurs séries $[1,3,4]$.

Les tumeurs des annexes cutanées sont des tumeurs rencontrées chez des sujets relativement jeunes $[1,5]$. La moyenne d'âge était de 39,75 ans. Samaïla retrouvait une moyenne d'âge de 33 ans [1].

Ces tumeurs se localisaient préférentiellement sur l'extrémité céphalique et les membres. En effet, la 
www.odermatol.com

Tableau 2 : Répartition des tumeurs bénignes pilaires en fonction de l'âge des patients $(n=22)$

\begin{tabular}{lcccc}
\hline Tranche d'âge (années) & Pilomatricome & Kyste trichilemmal proliférant & Trichoépithéliome & Total \\
\hline$<=10$ & 01 & 00 & 00 & 01 \\
{$[20-30]$} & 02 & 02 & 02 & 02 \\
{$[30-40]$} & 04 & 01 & 01 & 07 \\
{$[40-50]$} & 01 & 02 & 01 & 04 \\
{$[50-60]$} & 00 & 01 & 00 & 02 \\
{$[60-70]$} & 00 & 01 & 00 & 01 \\
{$[70-80]$} & 01 & 00 & 06 & 22 \\
Total & 09 & 07 & 01 \\
\hline
\end{tabular}

Le siège

Tableau 3 : Répartition des tumeurs bénignes pilaires en fonction du siège $(n=22)$

\begin{tabular}{lcccc}
\hline Siège anatomique & Pilomatricome & Kyste trichilemmal proliférant & Trichoépithéliome & Total \\
\hline Tête et cou & 06 & 05 & 05 & 16 \\
Tronc & 01 & 01 & 00 & 02 \\
Membres supérieurs & 01 & 01 & 01 & 00 \\
Membres inférieurs & 01 & 00 & 00 & 06 \\
Total & 09 & 07 & 01 \\
\hline
\end{tabular}

Tumeurs bénignes sébacées

L'âge

Tableau 4: Répartition des tumeurs bénignes sébacées en fonction de l'âge des patients $(n=14)$

\begin{tabular}{lcccc}
\hline $\begin{array}{l}\text { Tranche } \\
\text { d'âge } \\
\text { (années) }\end{array}$ & $\begin{array}{c}\text { Hamartome } \\
\text { verruco-sébacé }\end{array}$ & $\begin{array}{c}\text { Adénome } \\
\text { sébacé }\end{array}$ & $\begin{array}{c}\text { Hyperplasie } \\
\text { sébacée }\end{array}$ & Total \\
\hline$<=10$ & 01 & 00 & 00 & 01 \\
{$[10-20]$} & 01 & 01 & 00 & 02 \\
{$[20-30]$} & 01 & 00 & 01 & 02 \\
{$[30-40]$} & 02 & 01 & 01 & 04 \\
{$[40-50]$} & 01 & 01 & 01 & 03 \\
{$[50-60]$} & 01 & 00 & 00 & 01 \\
{$[60-70]$} & 01 & 00 & 00 & 01 \\
Total & 08 & 03 & 03 & 14 \\
\hline
\end{tabular}

Le siège

tête et le cou étaient les principales localisations des tumeurs pilaires et sèbacées. C'est un constat partagé par d'autres auteurs dont Samaïla (46\%), Sharma (64,28\%), Rajalakshm (10 localisations sur l'extrémité céphalique sur 21) [1,3-5]. Il s'agit en effet de zones anatomiquement riches en structures pilo-sébacées. Les tumeurs sudorales ont plus tendance à se localiser au niveau des extrémités et du tronc.

Les tumeurs à différenciation pilaire bénignes étaient les plus fréquentes; et dominées par le pilomatricome. Celui-ci représentait 17,3\% des tumeurs bénignes des annexes. Ce type histologique était également le plus représenté dans l'étude de Song et col [6]. Il s'agit d'une tumeur d'origine matricielle fréquente et facilement reconnaissable qui a la particularité unique d'aboutir à des cellules fantômes correspondant à une tentative abortive de produire une tige pilaire [5] (Fig. la). Cette tumeur a été décrite pour la première fois sous le nom “d'épithélioma momifié de Malherbe " en 1880 par

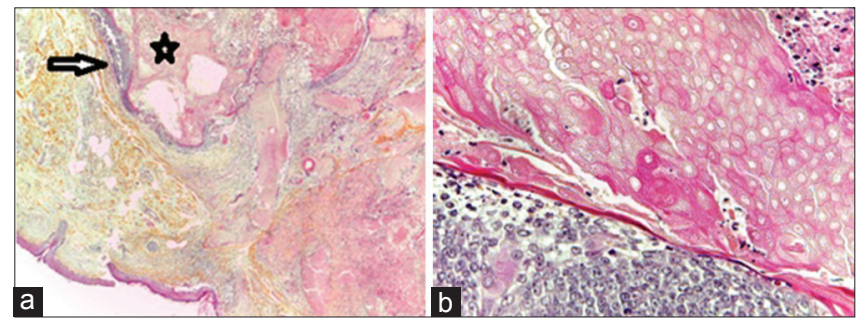

Figure 1: Pilomatricome Coloration HES. (a) G 40 Prolifération montrant une double cytologie constituée de cellules basophiles (flèche) et de cellules fantômes (étoile) séparées par une zone de transition (b) G100 Fort grossissement montrant les détails cytologiques et la zone de transition.

Source : Service d'anatomie pathologique/Centre Hospitalier Universitaire Yalgado Ouédraogo (CHU YO)

Dr Chenantals Malherbe [7]. Elle se rencontre à tout âge mais 40\% sont observés avant l'âge de 20 ans [8]. La grande majorité siège au niveau de l'extrémité céphalique (tête et cou) mais quelques-uns sont localisés sur les membres supérieurs et rarement le tronc [5].

Sur le plan histologique, on observe une prolifération dermique de plusieurs massifs de cellules, avec alternance de cellules fantômes anucléées réalisant un syncytium dont on voit très bien les membranes et de cellules matricielles très fortement basophiles, de petite taille à noyau central hyperchromatique (Fig. lb). Laspect pathognomonique est l'existence d'une zone de transition entre les cellules fantômes et les cellules basophiles [5].

C'est une tumeur bénigne pouvant subir des poussées de croissance très inquiétantes, surtout au départ, ou entrainer une réaction inflammatoire avec des douleurs. Lexérèse est curatrice [5]. 
www.odermatol.com

Tableau 5: Répartition des tumeurs bénignes sébacées en fonction du siège $(n=14)$

\begin{tabular}{lcccc}
\hline Siège anatomique & Hamartome verruco-sebacé & Adénome sebacé & Hyperplasie sébacée & Total \\
\hline Tête et cou & 06 & 03 & 03 & 12 \\
Tronc & 01 & 00 & 00 & 01 \\
Membres inférieurs & 01 & 00 & 00 & 01 \\
Total & 08 & 03 & 03 & 14 \\
\hline
\end{tabular}

Tumeurs bénignes sudorales

Les tumeurs bénignes eccrines en fonction de l'âge

Tableau 6: Répartition des tumeurs bénignes sudorales eccrines en fonction de l'âge $(n=12)$

\begin{tabular}{|c|c|c|c|c|c|c|}
\hline Type histologique & [20-30] & [30-40] & {$[40-50]$} & [50-60] & {$[60-70]$} & Total \\
\hline $\begin{array}{l}\text { Porome eccrine } \\
\text { classique }\end{array}$ & 00 & 03 & 01 & 01 & 01 & 06 \\
\hline Hidradénome nodulaire & 01 & 01 & 00 & 01 & 00 & 03 \\
\hline Syringome & 02 & 00 & 00 & 00 & 00 & 02 \\
\hline Cystadénome eccrine & 00 & 01 & 00 & 00 & 00 & 01 \\
\hline Total & 03 & 05 & 01 & 02 & 01 & 12 \\
\hline
\end{tabular}

Les tumeurs bénignes sudorales eccrines selon le siège

Tableau 7 : Répartition des tumeurs bénignes sudorales eccrines selon le siège $(n=12)$

\begin{tabular}{lccccc}
\hline Siège anatomique & Porome eccrine classique & Hidradénome nodulaire & Syringome & Cystadénome eccrine & Total \\
\hline Tête et cou & 02 & 00 & 01 & 01 & 04 \\
Tronc & 01 & 03 & 00 & 00 & 04 \\
Membres inférieurs & 03 & 00 & 00 & 00 \\
Membres supérieurs & 00 & 00 & 01 & 00 & 01 \\
Total & 07 & 03 & 02 & 01 \\
\hline
\end{tabular}

Les tumeurs bénignes sudorales apocrines selon l'âge et le siège

Les tumeurs bénignes sudorales étaient le deuxième groupe le plus rencontré. On distinguait les tumeurs eccrines et apocrines. Les tumeurs eccrines étaient les plus fréquentes localisées au niveau de l'extrémité céphalique mais aussi au niveau du tronc et des membres. Dans les séries de Samaïla, Rajalakshm et Sharma, ces tumeurs étaient les plus fréquentes [1-3]. Dans notre série, le porome eccrine classique était le type histologique prédominant. Il fait partie du groupe des poromes et est développé à partir des kératinocytes bordant le trajet intra épidermique du canal sudoripare eccrine. Il a été décrit pour la première fois en 1956 par Pinkus et col [9]. Il s'observe chez l'adulte à partir de la trentaine et siège le plus souvent au niveau des membres inférieurs ou au niveau des extrémités en particularité les régions palmo-plantaires [5]. La tumeur apparaît sous la forme d'une lésion saillante, bien limitée, à surface érythémateuse et parfois un peu suintante, attirant l'attention à l'examen clinique $[5,10]$. Sur le plan histologique, la tumeur est faite de grandes travées de petites cellules rondes formant des nappes très homogènes connectées à l'épiderme et descendant dans le derme moyen et profond (Fig. 2a). Il n'y a pas d'atypies cytologiques. On observe parfois de petits canaux (Fig. 2b) tantôt réduits à une lumière sans bordure, tantôt limités par un petit groupe de cellules porales avec ou sans cuticule éosinophile [5].

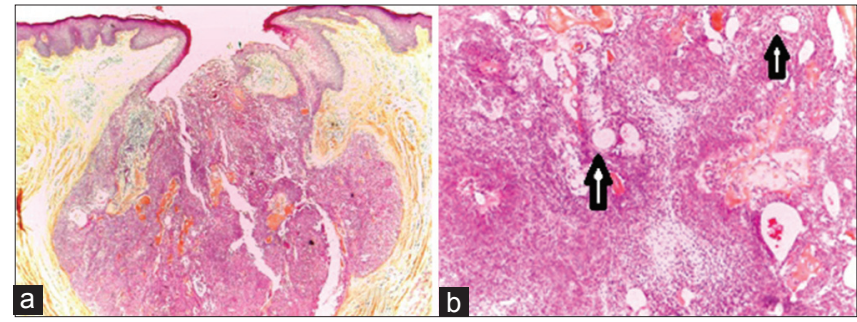

Figure 2 : Porome eccrine Coloration HES. (a) G 40 Prolifération en travées formant des nappes homogènes connectées à l'épiderme et s'enfonçant dans le derme moyen et profond, (b) G 100 Détail au fort grossissement montrant les formations canalaires au sein de la proliférationv(flèches).

Source : Service d'anatomie pathologique/Centre Hospitalier Universitaire Yalgado Ouédraogo (CHU YO)

Ces tumeurs doivent être excisées en totalité car les porocarcinomes surviennent dans près de la moitié des cas sur des poromes eccrines préexistants [5]. Le traitement est chirurgical.

Le troisième grand groupe est constitué des tumeurs bénignes à différenciation sébacée. Ces tumeurs viennent également au troisième rang dans la série de Rajalakshm et au deuxième rang dans la série de Samaïla et Sharma [1-3]. L'hamartome verrucosébacé était prédominant suivi de l'adénome sébacé. L'hamartome verruco-sébacé est plus une malformation, qu'un processus tumoral. C'est le plus complexe des 


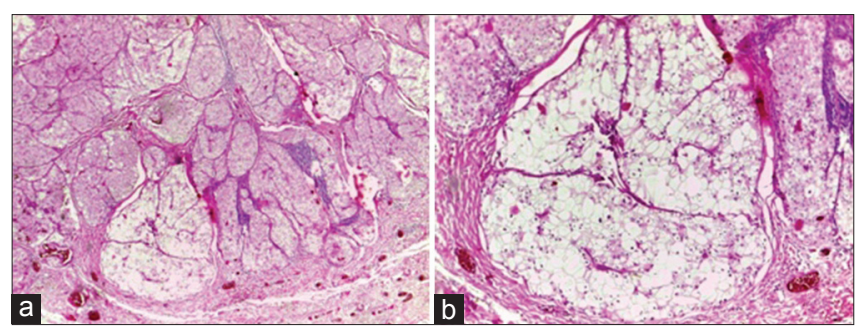

Figure $\mathbf{N}^{\circ} \mathbf{3}$ : Adénome sébacé Coloration HE (a) G 40 Prolifération réalisant de volumineux lobules sébacés associant en quantité variable des sébocytes matures et immatures (b) G100 Détail au fort grossissement montrant un volumineux lobule sébacé constitué de sébocytes matures.

Source : Service d'anatomie pathologique/Centre Hospitalier Universitaire Yalgado Ouédraogo (CHU YO)

hamartomes cutanés comprenant des éléments sébacés, glandulaires, pilaires et épidermiques. La grande majorité est localisée sur l'extrémité céphalique (visage, cuir chevelu). Il en existe au niveau du cou, du thorax et plus rarement des membres [5].

Laspect histologique évolue de la période néonatale à l'adolescence. En effet, à la période néo natale, on observe de volumineuses glandes sébacées superficielles ne semblant pas appendues à une structure folliculaire normale. On observe en surface un aspect lisse et une simple acanthose sans papillomatose ni hyperkératose. A la période de l'adolescence, l'aspect est verruqueux avec une acanthose endophytique qui rappelle une tumeur infundibulaire [5].

Ladénome sébacé est une tumeur bénigne localisée préférentiellement sur les zones riches en glandes sébacées (visage et cou) [5]. Il ne reproduit pas la structure normale de la glande, mais est fait de volumineux lobules sébacés associant en quantité variable des sébocytes matures et immatures (Figs. 3a et 3b). Les raisons esthétiques et fonctionnelles motivent l'exérèse de ces lésions. La transformation carcinomateuse est rare [5].

\section{CONCLUSION}

Les tumeurs des annexes de la peau sont rares, très souvent bénignes et localisées sur l'extrémité céphalique. Les raisons esthétiques et parfois fonctionnelles motivent l'exérèse de ces lésions. Cette exérèse dans la plupart des cas est curatrice. Certaines de ces tumeurs bénignes ont un potentiel de dégénérescence maligne d'où la nécessité d'une exérèse suivie d'un examen anatomopathologique.

\section{RÉFÉRENCES}

1. Samaila M. Adnexal skin tumor in Zaria, Nigeria. Ann Afr Med 2008; 7: 7-10.

2. Rajalakshm V, Selvakumar S, Rajeswari K, Meenakshisundaram K, Veena G, Ramachandran P. Case serie of Skin Adnexal Tumours. J Clin Diag Res. 2014;8:FC07-10.

3. Sharma A, Paricharak DP, Nigam JS, Rewri S, Soni PB, Omhare A, et al. Histopathological Study of Skin Adnexal Tumours Institutional Study in South India. J Skin Cancer. 2014;2014:543756.

4. Radhika K, Phaneendra BV, Rukmangadha N, Reddy MK. A study of biopsy confirmed skin adnexal tumors: experience at a tertiary care teaching hospital. J Clin Scien Res. 2013;7:132-8.

5. Wechsler J. Pathologie cutanée tumorale. Editeur: Sauramp médical (Montpellier), 2009.

6. Song KY, Yoon DH, Ham EK, Lee SY. Clinicopathological study on the appendage tumors. Korean J Pathol. 1989;23:111-21.

7. Jocopo N, Dalmar A, Elisabetta G, Massimo C. Pilomatrixoma of the breast, a rare lesion simulating breast cancer: a case report. Radiol Case. 2013;7:43-50.

8. Cupta R, Verma S, Bansal P, Moha A. Pilomatrixoma of the arm: a rare case with cytologic diagnosis. Dermatol Med. 2012;10:3p.

9. Goldman P, Pinkus H, Rogin J R. Eccrine Poroma: tumors exhibiting feature of the epidermal sweat duct unit. AMA Arch Derm. 1956;74:511-21.

10. Avilés-Izquierdo JA, Valazquez-Targuelo D, Lecona-Echevarrie M, Lazare-Ochaitu P. Caracteristicas dermoscopia del poroma ecrino. Acta Dermosifiligor. 2009;100:133-6.

Copyright by Aimé Sosthène Ouédraogo, et al. . This is an open-access article distributed under the terms of the Creative Commons Attribution License, which permits unrestricted use, distribution, and reproduction in any medium, provided the original author and source are credited.

Source of Support: Nil, Conflict of Interest: None declared. 